\title{
UNIFORM APPROXIMATIONS TO A CLASS OF BESSEL FUNCTIONS
}

\section{ALFRED M. PEISER}

1. Consider a function $t(x, y)$, defined in $x \geqq 0, y \geqq 0$, with continuous first partial derivatives, which satisfies the differential equation

$$
\frac{\partial^{2} t}{\partial x \partial y}+\frac{\partial t}{\partial x}+\frac{\partial t}{\partial y}=0 .
$$

It is well known ${ }^{1}$ that $t(x, y)$ is uniquely determined when boundary values $t(x, 0)$ and $t(0, y)$ are specified. By a straight-forward application of Laplace transforms, one may obtain (formally) the solution

$$
\begin{aligned}
t(x, y)= & t(x, 0) e^{-y}+\int_{0}^{x} t(u, 0) K_{1}(x-u, y) d u \\
& +\int_{0}^{y}\left[t(0, v)+\frac{\partial t(0, v)}{\partial v}\right] K_{0}(y-v, x) d v,
\end{aligned}
$$

where

$$
K_{n}(x, y)=(y / x)^{n / 2} e^{-x-y} I_{n}\left(2(x y)^{1 / 2}\right), \quad n=0,1,2, \cdots,
$$

and $I_{n}$ is the Bessel function of order $n$ and imaginary argument. It is not difficult to verify, by direct differentiation, that (2) satisfies (1).

In certain applications, ${ }^{2} t(0, y)$ is given explicitly but $t(x, 0)$ is given in terms of the values of $t(x, y)$ at some other (fixed) value of $y$, namely,

$$
t(x, 0)=a+b t(x, P) \text { or } t(x, 0)=a+b t(S-x, P),
$$

where $a, b, P$, and $S$ are constants. In such cases, one readily obtains, from (2), an integral equation for $t(x, 0)$ of the form

$$
\left.\begin{array}{r}
t(x, 0) \\
t(S-x, 0)
\end{array}\right\}=F(x)+b t(x, 0) e^{-P}+b \int_{0}^{x} t(u, 0) K_{1}(x-u, P) d u,
$$

Presented to the Society, April 30, 1949; received by the editors April 9, 1949 and, in revised form, August 8, 1949. 21.

$1 \mathrm{~J}$. D. Tamarkin, Partial differential equations, Brown University Notes, 1941, p.

'See for example, H. Hausen, Naherungsverfahren zur Berechnung des Warmeaustausches in Regeneratoren, Zeitschrift für Angewandte Mathematik und Mechanik vol. 11 (1931) pp. 105-114. 
where $F(x)$ is known.

In the evaluation of (2) for particular boundary conditions, ${ }^{8}$ or in the solution of integral equations of the type (4), it is sometimes convenient to replace $K_{0}(x, y)$ and $K_{1}(x, y)$ by approximating functions which are more suitable for numerical or analytical work. For large values of the arguments, one could, of course, use the asymptotic formulas for the Bessel functions, ${ }^{4}$ while for small values of the arguments, the power series expansions would suffice. It is desirable, however, particularly in the case of the integral equation (4), to obtain approximations to $K_{0}(x, y)$ and $K_{1}(x, y)$ which hold uniformly in $0 \leqq x<\infty$.

We shall show that, for suitably large values of $y, K_{0}$ and $K_{1}$ can be approximated uniformly in $x$ by means of the normal probability function,

$$
\Phi(x)=\frac{1}{(2 \pi)^{1 / 2}} e^{-x^{2} / 2}
$$

We find that

$$
\lim _{y \rightarrow \infty}(2 y+1)^{1 / 2} K_{0}\left(y+1+x(2 y+1)^{1 / 2}, y\right)=\Phi(x)
$$

and

$$
\lim _{y \rightarrow \infty}(2 y)^{1 / 2} K_{1}\left(y+x(2 y)^{1 / 2}, y\right)=\Phi(x),
$$

and obtain, for $x \geqq 0$, the inequalities

$$
\begin{array}{ll} 
& \left|K_{0}(x, y)-\frac{1}{(2 y+1)^{1 / 2}} \Phi\left(\frac{x-y-1}{(2 y+1)^{1 / 2}}\right)\right| \\
\quad<\frac{0.321}{2 y+1}+\frac{5.101}{(2 y+1)^{3 / 2}}+\frac{0.894}{(2 y+1)^{1 / 2}} \sigma^{-y / 5}, \quad y \geqq 10, \\
\left|K_{1}(x, y)-\frac{1}{(2 y)^{1 / 2}} \Phi\left(\frac{x-y}{(2 y)^{1 / 2}}\right)\right| & \\
\quad<\frac{0.167}{y}+\frac{0.800}{y^{3 / 2}}+\frac{0.694}{y^{1 / 2}} e^{-y / 5}, & y \geqq 10 .
\end{array}
$$

Examination of tabulated values of the functions indicates that

See, for example, P. Bateman, Partial differential equations, Dover, 1944, p. 125; A. M. Peiser, The unsteady state cooling of beds, submitted for publication to Industrial and Engineering Chemistry.

1 G. N. Watson, Bessel functions, Cambridge, 1945, p. 203. 
these error estimates exceed the actual errors by at most about a factor of two. Better approximations to $K_{0}$ and $K_{1}$ may be obtained by means of asymptotic series involving the successive derivatives of $\Phi(x)$. Series of this type are given in \$6.

2. The Laplace transforms. We define $K_{n}(x, y)$ to be zero when $x<0$ and consider the bilateral Laplace transforms

$$
\begin{aligned}
& k_{n}(\lambda, y)=\int_{-\infty}^{\infty} e^{-\lambda x} K_{n}(x, y) d x, \quad R \lambda>-1, \\
& \phi(\lambda)=\int_{-\infty}^{\infty} e^{-\lambda x} \Phi(x) d x=e^{\lambda^{2} / 2} .
\end{aligned}
$$

By standard integration formulas, ${ }^{5}$ we find

Let

$$
\begin{aligned}
& k_{0}(\lambda, y)=\exp \left\{-y+\frac{y}{\lambda+1}\right\} /(\lambda+1), \quad R \lambda>-1, \\
& k_{1}(\lambda, y)=\exp \left\{-y+\frac{y}{\lambda+1}\right\}-\exp \{-y\}, \quad R \lambda>-1 .
\end{aligned}
$$

(9a)

$$
\begin{array}{rrr}
\text { (9a) } H_{0}(x, y)=(2 y+1)^{1 / 2} K_{0}\left(y+1+x(2 y+1)^{1 / 2}, y\right), & x \geqq-\frac{y+1}{(2 y+1)^{1 / 2}}, \\
\text { (9b) } \quad H_{1}(x, y)=(2 y)^{1 / 2} K_{1}\left(y+x(2 y)^{1 / 2}, y\right), & x \geqq-(y / 2)^{1 / 2} .
\end{array}
$$

Then, if $h_{n}(\lambda, y)$ denotes the bilateral transform of $H_{n}(x, y)$, we have $\mathrm{e}^{\mathrm{b}}$

$$
h_{0}(\lambda, y)=k_{0}\left(\lambda /(2 y+1)^{1 / 2}, y\right) \exp \left\{\frac{\lambda(y+1)}{(2 y+1)^{1 / 2}}\right\}
$$

$$
\begin{aligned}
= & \exp \left\{-y+\frac{y}{1+\lambda /(2 y+1)^{1 / 2}}\right. \\
+ & \left.\frac{\lambda(y+1)}{(2 y+1)^{1 / 2}}\right\} /\left(1+\lambda /(2 y+1)^{1 / 2}\right), \\
h_{1}(\lambda, y)= & k_{1}\left(\lambda /(2 y)^{1 / 2}, y\right) \exp \left\{\lambda(y / 2)^{1 / 2}\right\} \\
= & \exp \left\{-y+\lambda(y / 2)^{1 / 2}\right\} \\
& \cdot\left[\exp \left\{\frac{y}{1+\lambda /(2 y)^{1 / 2}}\right\}-1\right] .
\end{aligned}
$$

- R. V. Churchill, Modern operational mathematics in engineering, New York, 1944, pp. 294, 299, formula 81. 
It follows from (8) that

$$
\lim _{y \rightarrow \infty} h_{n}(\lambda, y)=\phi(\lambda), \quad n=0,1,
$$

uniformly in each bounded region of the complex $\lambda$-plane. From this result and from standard theorems for the Laplace transform, one can deduce (6a) and (6b). It is not necessary to give details at this point since we are going to prove the stronger results (7a) and (7b).

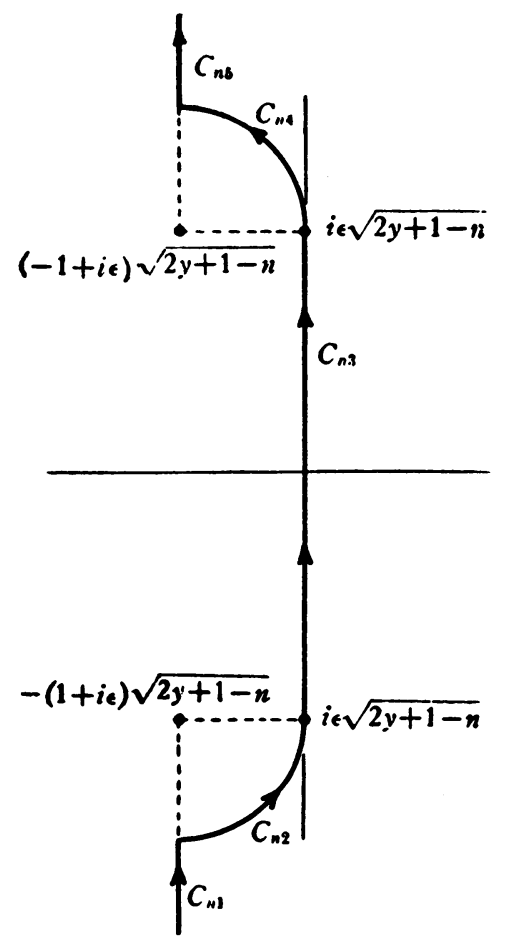

Fig. 1

By means of the complex inversion formula for the Laplace transform, ${ }^{6}$ we can write

$H_{n}(x, y)=\frac{1}{2 \pi i} \int_{C_{n}} e^{\lambda x} h_{n}(\lambda, y) d \lambda, \quad x \geqq-\frac{y+1-n}{(2 y+1-n)^{1 / 2}}, n=0,1$,

where $C_{n}$ is any vertical line $R \lambda=\alpha$, with $\alpha>-(2 y+1-n)^{1 / 2}$, and where the principal value of the integral is to be taken. Now, $h_{n}(\lambda, y)$

- D. V. Widder, The Laplace transform, Princeton, 1946, p. 241. 
is analytic at each point of the finite $\lambda$-plane except for an essential singularity at $\lambda=-(2 y+1-n)^{1 / 2}$. By a simple application of Cauchy's theorem, we can replace the contour $C_{n}$ by the contour shown in Fig. 1. We assume $0<\epsilon<1$. If we let

$$
I_{n j}=\frac{1}{2 \pi i} \int_{C_{n j}} e^{\lambda x} h_{n}(\lambda, y) d \lambda,
$$

we can write, for $n=0,1$, and $x \geqq-(y+1-n) /(2 y+1-n)^{1 / 2}$,

$$
\begin{aligned}
\mid H_{n}(x, y) & -\Phi(x) \mid \\
& \leqq\left|I_{n 3}-\Phi(x)\right|+\left|I_{n 1}+I_{n b}\right|+\left|I_{n 2}+I_{n 4}\right| .
\end{aligned}
$$

In the following sections we obtain upper bounds for each of the quantities on the right-hand side of (12).

3. Preliminary lemmas. We first prove the following lemmas:

Lemma 1. If $a>0,-\epsilon<u<\epsilon<1$, then

$$
\exp \left\{-\frac{a}{1+i u}\right\}=e^{-a}+i a u \epsilon^{-a}+\frac{\theta u^{2}}{1-\epsilon} e^{-a / 2}, \quad|\theta|<1 .
$$

Lemma 2. If $b>0,-\epsilon<u<\epsilon<1$, then

$$
\begin{aligned}
f(i u)= & \frac{1}{1+i u} \exp \left\{\frac{1+i u}{2}-\frac{b+1}{2(1+i u)}\right\}=e^{-b / 2}+\frac{i b u}{2} e^{-b / 2} \\
& +\frac{\theta u^{2} e^{-b / 4}}{\pi(1-\epsilon)}\left[\frac{e^{z / 4}}{(2 \epsilon)^{1 / 2}} \arccos (\epsilon-1)+\frac{8 e^{\epsilon / 2-1 / 4}}{b(2-\epsilon)}\right], \quad|\theta|<1 .
\end{aligned}
$$

To prove Lemma 1, we apply Taylor's theorem in the form

$$
\begin{aligned}
& \exp \left\{-\frac{a}{1+i u}\right\} \\
& \quad=e^{-a}+i a u e^{-a}-\frac{u^{2}}{2 \pi i} \int_{c} \frac{\exp \{-a /(1+w)\}}{w^{2}(w-i u)} d w,
\end{aligned}
$$

where $C$ is the circle $|w|=\rho, \epsilon<\rho<1$. For $w$ on $C, w=\rho e^{i \phi}$, we have

$$
\left|\frac{\exp \{-a /(1+w)\}}{w^{2}(w-i u)}\right| \leqq \frac{\exp \{-a R(1 /(1+w))\}}{\rho^{2}(\rho-\epsilon)}, \quad R=\text { real part. }
$$

But, for $\rho<1$,

$$
R \frac{1}{1+w}=\frac{1+\rho \cos \phi}{1+\rho^{2}+2 \rho \cos \phi} \geqq \frac{1}{1+\rho},
$$


so that

$$
\left|\frac{\exp \{-a /(1+w)\}}{w^{2}(w-i u)}\right| \leqq \frac{\exp \{-a /(1+\rho)\}}{\rho^{2}(\rho-\epsilon)} .
$$

Lemma 1 now follows easily from (13) if we let $\rho \rightarrow 1$.

To prove Lemma 2, we apply Taylor's theorem in the form

$$
f(i u)=e^{-b / 2}+\frac{i b u}{2} e^{-b / 2}-\frac{u^{2}}{2 \pi i} \int_{\mathrm{r}} \frac{f(w)}{w^{2}(w-i u)} d w .
$$

where $\Gamma$ is the contour shown in Fig. 2. We note that for $w$ on $\Gamma$,

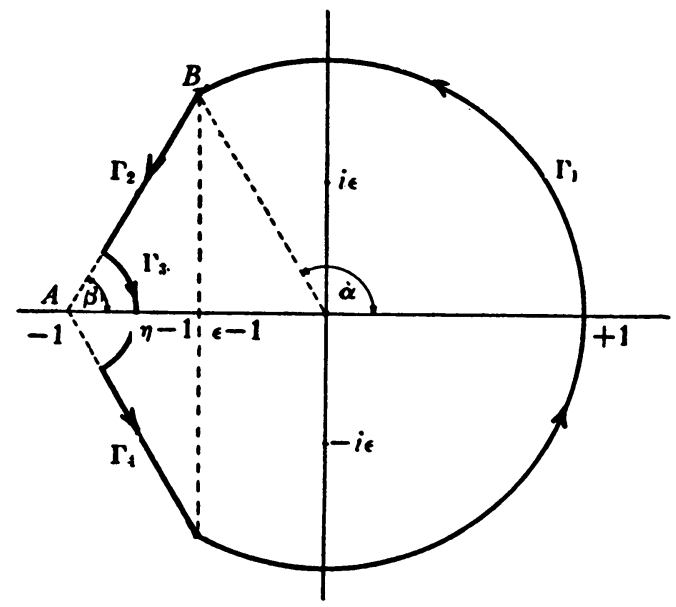

Fig. 2

$|w-i u|>1-\epsilon$, and that $A B=(2 \epsilon)^{1 / 2}, \cos \beta=(\epsilon / 2)^{1 / 2}, \cos \alpha=\epsilon-1$.

For $w$ on $\Gamma_{1}, w=e^{i \phi}, \cos \phi>\epsilon-1$, and we have

$$
\begin{aligned}
|f(w)| & =(2+2 \cos \phi)^{-1 / 2} \exp \left\{\frac{1+\cos \phi}{2}-\frac{b+1}{2} R \frac{1}{1+e^{i \phi}}\right\} \\
& \leqq \frac{1}{(2 \epsilon)^{1 / 2}} \exp \left\{1-\frac{b+1}{4}\right\} .
\end{aligned}
$$

Hence

$$
\left|\frac{1}{2 \pi i} \int_{\mathrm{r}_{1}} \frac{f(w) d w}{w^{2}(w-i u)}\right| \leqq \frac{e^{3 / 4-b / 4}}{\pi(2 \epsilon)^{1 / 2}(1-\epsilon)} \operatorname{arc} \cos (\epsilon-1) .
$$

For $w$ on $\Gamma_{2}, w=-1+r e^{i \beta},|w|^{2}>1-\epsilon / 2, \eta<r<(2 \epsilon)^{1 / 2}$, and 


$$
\begin{aligned}
|f(w)| & =\frac{1}{r} \exp \left\{\frac{r}{2} \cos \beta-\frac{b+1}{2 r} \cos \beta\right\} \\
& <\frac{1}{r} \exp \left\{\frac{\epsilon}{2}-\frac{b+1}{2 r}\left(\frac{\epsilon}{2}\right)^{1 / 2}\right\} .
\end{aligned}
$$

Thus

$$
\begin{aligned}
\left|\frac{1}{2 \pi i} \int_{\Gamma_{2}} \frac{f(w) d w}{w^{2}(w-i u)}\right| \\
\quad \leqq \frac{e^{\epsilon / 2}}{\pi(1-\epsilon)(2-\epsilon)} \int^{(2 \epsilon)^{1 / 2}} \frac{1}{r} \exp \left\{-\frac{b+1}{2 r}\left(\frac{\epsilon}{2}\right)^{1 / 2}\right\} d r \\
\quad<\frac{4 e^{\epsilon / 2-(b+1) / 4}}{\pi(1-\epsilon)(2-\epsilon) b} .
\end{aligned}
$$

The same estimate holds for the integral over $\Gamma_{4}$. It is easy to show

$$
\left|\frac{1}{2 \pi i} \int_{\Gamma_{3}} \frac{f(w)}{w^{2}(w-i u)} d w\right| \rightarrow 0 \quad \text { as } \eta \rightarrow 0 .
$$

Combining (14), (15), (16), and (17), we obtain Lemma 2.

4. Error estimate for $K_{1}$. To obtain the estimate (7b), we note first, from (10b), (11), and Fig. 1, that

$$
\begin{aligned}
I_{13}=\frac{1}{2 \pi} \int_{-\epsilon(2 y)^{1 / 8}}^{e(2 y)^{1 / 2}} e^{i v x} h_{1}(i v, y) d v & \\
=\frac{1}{2 \pi} \int_{-\epsilon(2 y)^{1 / 2}}^{e(2 y)^{1 / 2}} e^{i v x}\left[\exp \left\{-\frac{v^{2}}{2} \cdot \frac{1}{1+i v /(2 y)^{1 / 2}}\right\}\right. & \left.-\exp \left\{-y+i v(y / 2)^{1 / 2}\right\}\right] d v .
\end{aligned}
$$

We apply Lemma 1 with $a=v^{2} / 2, u=v /(2 y)^{1 / 2}$, to obtain

$$
\begin{aligned}
I_{13}=\frac{1}{2 \pi} \int_{-\epsilon(2 y)^{1 / 2}}^{e(2 y)^{1 / 2}} e^{i v x}\left[e^{-v^{2} / 2}\right. & +\frac{i v^{3}}{2(2 y)^{1 / 2}} e^{-v^{2} / 2} \\
& \left.+\frac{\theta v^{2}}{2 y(1-\epsilon)} e^{-v^{2} / 4}-e^{-y+i v(\nu / 2)^{1 / 2}}\right] d v .
\end{aligned}
$$

Combining (5) and (8), we have

$$
\Phi(x)=\frac{1}{2 \pi} \int_{-\infty}^{\infty} e^{i v x-v^{2} / 2} d v,
$$


and we can write

$$
\begin{aligned}
\left|I_{18}-\Phi(x)\right| \leqq & \frac{1}{2 \pi} \int_{|v|>\epsilon(2 y)^{1 / 2}} e^{-v^{2} / 2} d v+\frac{1}{4 \pi(2 y)^{1 / 2}} \int_{-\infty}^{\infty}|v|^{8} e^{-v^{2} / 2} d v \\
& +\frac{1}{4 \pi y(1-\epsilon)} \int_{-\infty}^{\infty} v^{2} e^{-v^{2} / 4} d v+\frac{\epsilon(2 y)^{1 / 2}}{\pi} e^{-\nu}
\end{aligned}
$$

With the inequality

$$
\int_{|v|>\delta} e^{-v^{2} / 2} d v<\frac{1}{\delta} \int_{|v|>\delta} v e^{-v^{2} / 2} d v=\frac{2}{\delta} e^{-\delta^{2} / 2}
$$

we find

$$
\text { (19) }\left|I_{18}-\Phi(x)\right| \leqq \frac{1}{\pi(2 y)^{1 / 2}}+\frac{1}{\pi^{1 / 2}(1-\epsilon) y}+\frac{e^{-\epsilon^{2} y}}{\pi \epsilon(2 y)^{1 / 2}}+\frac{\epsilon(2 y)^{1 / 2}}{\pi} e^{-y} \text {. }
$$

For the integrals $I_{11}$ and $I_{16}$ we make the change of variables $\lambda=(2 y)^{1 / 2}(-1+i v)$. From (10b) and (11) we obtain

$$
I_{11}+I_{16}=\frac{(2 y)^{1 / 2}}{2 \pi} e^{-2 y-x(2 y)^{1 / 2}} \int_{|v|>1+c} e^{i v\left(y+x(2 y)^{1 / 2}\right.}\left[e^{-i y / v}-1\right] d v .
$$

For real $u$, we can write $e^{i u}=1+i u+\theta u^{2} / 2,|\theta|<1$. Hence

$$
I_{11}+I_{15}=\frac{(2 y)^{1 / 2}}{2 \pi} e^{-2 y-x(2 y)^{1 / 2}} \int_{|v|>1+e} e^{i v\left(y+x(2 y)^{1 / 2}\right)}\left[-\frac{i y}{v}+\frac{\theta y^{2}}{2 v^{2}}\right] d v .
$$

Since $x \geqq-(y / 2)^{1 / 2}$, we have

$$
\begin{aligned}
\left|I_{11}+I_{16}\right| \leqq \frac{(2 y)^{1 / 2}}{2 \pi} e^{-y}\left[\left|\int_{|v|>1+e} y e^{i v\left(v+x(2 v)^{1 / 2}\right)} \frac{d v}{v}\right|\right. & \\
& \left.+\frac{y^{2}}{2} \int_{|v|>1+e} \frac{d v}{v^{2}}\right] .
\end{aligned}
$$

But for $\gamma>0$,

$$
\left|\int_{|v|>\gamma} e^{i v} \frac{d v}{v}\right|=\left|\int_{|v|>\gamma} \frac{\sin v}{v} d v\right|<\pi,
$$

so that

$$
\left|I_{11}+I_{1 b}\right| \leqq \frac{(2 y)^{1 / 2}}{2 \pi} \epsilon^{-y}\left\{\pi y+\frac{y^{2}}{1+\epsilon}\right\} .
$$

To estimate $I_{14}$, let $\lambda=(2 y)^{1 / 2}\left(-1+i \epsilon+e^{i \phi}\right)$. Then 


$$
\begin{aligned}
I_{14}=\frac{(2 y)^{1 / 2}}{2 \pi} e^{-y} \int_{0}^{\pi / 2} \exp \left\{\left(y+x(2 y)^{1 / 2}\right)\left(-1+i \epsilon+e^{i \phi}\right)\right\} \\
\cdot\left[e^{y /\left(i \epsilon+e^{i \phi}\right)}-1\right] e^{i \phi} d \phi .
\end{aligned}
$$

Since $x \geqq-(y / 2)^{1 / 2}$,

$$
\begin{aligned}
\left|I_{14}\right| & \leqq \frac{(2 y)^{1 / 2}}{2 \pi} e^{-y} \int_{0}^{\pi / 2} \exp \left\{\left(y+x(2 y)^{1 / 2}\right)(\cos \phi-1)\right\} \\
\cdot\left[1+\exp \left\{\frac{y \cos \phi}{1+\epsilon^{2}+2 \epsilon \sin \phi}\right\}\right] d \phi & \leqq \frac{(2 y)^{1 / 2}}{2 \pi} \epsilon^{-y} \int_{0}^{\pi / 2}\left[1+\exp \left\{\frac{y \cos \phi}{1+\epsilon^{2}}\right\}\right] d \phi .
\end{aligned}
$$

With the change of variable $\sin (\phi / 2)=u$, we find

$$
\begin{aligned}
\int_{0}^{\pi / 2} \exp & \left\{\frac{y \cos \phi}{1+\epsilon^{2}}\right\} d \phi \\
= & 2 \exp \left\{\frac{y}{1+\epsilon^{2}}\right\} \int_{0}^{1 / 2^{1 / 2}} \frac{\exp \left\{-2 y u^{2} /\left(1+\epsilon^{2}\right)\right\} d u}{\left(1-u^{2}\right)^{1 / 2}} \\
& <2^{8 / 2} \exp \left\{\frac{y}{1+\epsilon^{2}}\right\} \int_{0}^{\infty} \exp \left\{\frac{-2 y u^{2}}{1+\epsilon^{2}}\right\} d u \\
& =\left(\frac{\pi\left(1+\epsilon^{2}\right)}{y}\right)^{1 / 2} \exp \left\{\frac{y}{1+\epsilon^{2}}\right\} .
\end{aligned}
$$

Thus,

$$
\left|I_{14}\right| \leqq \frac{(2 y)^{1 / 2}}{4} e^{-y}+\left(\frac{1+\epsilon^{2}}{2 \pi}\right)^{1 / 2} \exp \left\{-\frac{\epsilon^{2} y}{1+\epsilon^{2}}\right\}
$$

The same estimate may be obtained for $I_{12}$.

To obtain (7b), we take $y \geqq 10$ and $\epsilon=1 / 2$. Then (19), (20), and (22) become, respectively,

$$
\begin{aligned}
\left|I_{13}-\Phi(x)\right| & \leqq \frac{1}{\pi(2 y)^{1 / 2}}+\frac{2}{\pi^{1 / 2} y}+\frac{2^{1 / 2}}{\pi y^{1 / 2}} e^{-y / 4}+\frac{y^{1 / 2}}{\pi 2^{1 / 2}} e^{-y} \\
& <\frac{1}{\pi(2 y)^{1 / 2}}\left(1+y e^{-y}\right)+\frac{2}{\pi^{1 / 2} y}+\frac{1}{\pi}\left(\frac{2}{y e}\right)^{1 / 2} e^{-y / 5} \\
& <\frac{0.2252}{y^{1 / 2}}+\frac{1.130}{y}+0.0864 e^{-y / 5}
\end{aligned}
$$




$$
\begin{aligned}
\left|I_{11}+I_{15}\right| & \leqq \frac{1}{\pi(2 y)^{1 / 2}} e^{-y}\left(\pi y^{2}+\frac{2}{3} y^{z}\right)<\frac{0.0101}{y^{1 / 2}} \\
\left|I_{12}\right|+\left|I_{14}\right| & \leqq \frac{1}{(2 y)^{1 / 2}} \cdot y e^{-y}+\left(\frac{5}{2 \pi}\right)^{1 / 2} e^{-y / 5} \\
& <\frac{0.0004}{y^{1 / 2}}+0.893 e^{-y / 5}
\end{aligned}
$$

Combining (12), (23), (24), and (25), we obtain finally

$$
\left|H_{1}(x, y)-\Phi(x)\right|<\frac{0.236}{y^{1 / 2}}+\frac{1.130}{y}+0.980 e^{-y / 5} \text {. }
$$

Equation (7b) now follows from (9b) and this inequality.

5. Error estimate for $K_{0}$. The procedure used to obtain (7b) may be followed with obvious modifications to obtain (7a). Accordingly, we shall only sketch the proof of (7a). From (10a), (11), and Fig. 1, we have

$$
\begin{aligned}
I_{03}=\frac{1}{2 \pi} \int_{-\epsilon(2 y+1)^{1 / 2}}^{e(2 y+1)^{1 / 2}} e^{i v x} h_{0}(i v, y) d v & \\
=\frac{1}{2 \pi} \int_{-\epsilon(2 y+1)^{1 / 2}}^{e(2 y+1)^{1 / 2}} e^{i v x} \exp & \left\{-\frac{v^{2}+1}{2\left(1+i v /(2 y+1)^{1 / 2}\right)}\right. \\
& \left.+\frac{1+i v /(2 y+1)^{1 / 2}}{2}\right\} \frac{d v}{1+i v /(2 y+1)^{1 / 2}} .
\end{aligned}
$$

We apply Lemma 2 with $b=v^{2}, u=v /(2 y+1)^{1 / 2}$, to obtain

$$
\begin{aligned}
I_{03}= & \frac{1}{2 \pi} \int_{-\epsilon(2 y+1)^{1 / 2}}^{\epsilon(2 y+1)^{1 / 2}} e^{i v x}\left[e^{-v^{2} / 2}+\frac{i v^{8}}{2(2 y+1)^{1 / 2}} e^{-v^{2} / 2}\right. \\
& \left.+\frac{\theta e^{-v^{2} / 4}}{\pi(2 y+1)(1-\epsilon)}\left(\frac{e^{8 / 4}}{(2 \epsilon)^{1 / 2}} \arccos (\epsilon-1) \cdot v^{2}+\frac{8 e^{\epsilon / 2-1 / 4}}{2-\epsilon}\right)\right] d v .
\end{aligned}
$$

Proceeding as in $\$ 4$, we find

$$
\begin{aligned}
\mid I_{03} & -\Phi(x) \mid<\frac{1}{\pi(2 y+1)^{1 / 2}}+\frac{\exp \left\{-\left(\epsilon^{2} / 2\right)(2 y+1)\right\}}{\pi \epsilon(2 y+1)^{1 / 2}} \\
& +\frac{2}{\pi^{8 / 2}(2 y+1)(1-\epsilon)}\left[\frac{e^{8 / 4}}{(2 \epsilon)^{1 / 2}} \operatorname{arc~cos}(\epsilon-1)+\frac{4 e^{\epsilon / 2-1 / 4}}{(2-\epsilon)}\right] .
\end{aligned}
$$

For the integrals $I_{01}$ and $I_{05}$, we make the change of variables 
$\lambda=(2 y+1)^{1 / 2}(-1+i v)$ and obtain

$$
\begin{aligned}
I_{01}+I_{06}=\frac{(2 y+1)^{1 / 2}}{2 \pi i} \exp \left\{-y-\left(y+1+x(2 y+1)^{1 / 2}\right)\right\} & \\
& \int_{|v|>1+e} e^{i v\left(y+1+x(2 y+1)^{1 / 2}\right)}\left(1+\frac{\theta y}{v}\right) \frac{d v}{v},
\end{aligned}
$$

with $|\theta|<1$. Since $x \geqq-(y+1) /(2 y+1)^{1 / 2}$, we find, as in $\$ 4$,

$$
\left|I_{01}+I_{05}\right|<\frac{e^{-y}(2 y+1)^{1 / 2}}{2 \pi}\left(\pi+\frac{2 y}{1+\epsilon}\right) .
$$

For $I_{04}$ (and, similarly for $\left.I_{02}\right)$, we let $\lambda=(2 y+1)^{1 / 2}\left(-1+i \epsilon+e^{i \phi}\right)$, so that

$$
\begin{array}{r}
I_{04}=\frac{(2 y+1)^{1 / 2}}{2 \pi} e^{-y} \int_{0}^{\pi / 2} \exp \{(y+1+ \\
\left.+\frac{\left.y(2 y+1)^{1 / 2}\right)\left(-1+i \epsilon+e^{i \phi}\right)}{i \epsilon+e^{i \phi}}+i \phi\right\} \frac{d \phi}{i \epsilon+e^{i \phi}},
\end{array}
$$

and

$$
\begin{aligned}
\left|I_{04}\right| \leqq \frac{(2 y+1)^{1 / 2}}{2 \pi} e^{-\nu} \int_{0}^{\pi / 2} \exp \left\{\left(y+1+x(2 y+1)^{1 / 2}\right)(\cos \phi-1)\right. \\
\left.+\frac{y \cos \phi}{1+\epsilon^{2}+2 \epsilon \sin \phi}\right\} \frac{d \phi}{\left(1+\epsilon^{2}+2 \epsilon \sin \phi\right)^{1 / 2}} \\
<\frac{(2 y+1)^{1 / 2}}{2 \pi\left(1+\epsilon^{2}\right)^{1 / 2}} e^{-\nu} \int_{0}^{\pi / 2} \exp \left\{\frac{y \cos \phi}{1+\epsilon^{2}}\right\} d \phi .
\end{aligned}
$$

Using (21), we obtain

$$
\left|I_{04}\right|<\frac{(2 y+1)^{1 / 2}}{(2 \pi)^{1 / 2}(2 y)^{1 / 2}} \exp \left\{-\frac{\epsilon^{2} y}{1+\epsilon^{2}}\right\} .
$$

If we take $y \geqq 10$ and $\epsilon=1 / 2$, the inequalities (26), (27), and (28) become, respectively,

$$
\begin{aligned}
\left|I_{03}-\Phi(x)\right|< & \frac{1}{\pi(2 y+1)^{1 / 2}}+\frac{4}{\pi^{3 / 2}(2 y+1)}\left[\frac{2 \pi e^{3 / 4}}{3}+\frac{8}{3}\right] \\
& +\frac{2 e^{-1 / 8-y / 4}}{\pi(2 y+1)^{1 / 2}} \\
& <\frac{0.3184}{(2 y+1)^{1 / 2}}+\frac{5.101}{2 y+1}+0.0735 e^{-y / 5}
\end{aligned}
$$




$$
\begin{aligned}
\left|I_{01}+I_{05}\right|<\frac{(2 y+1) e^{-y}(\pi+4 y / 3)}{2 \pi(2 y+1)^{1 / 2}}<\frac{0.0025}{(2 y+1)^{1 / 2}} & \\
\left|I_{02}\right|+\left|I_{14}\right| & <0.820 e^{-y / 5}
\end{aligned}
$$

Combining (12), (29), (30), and (31), we obtain

$$
\left|H_{0}(x, y)-\Phi(x)\right|<\frac{0.321}{(2 y+1)^{1 / 2}}+\frac{5.101}{2 y+1}+0.894 e^{-y / 5} .
$$

Equation (7a) now follows from (9a) and the last inequality.

6. Series expansion for $K_{n}(x, y)$. Each of the functions $K_{n}(x, y)$ can be expanded in terms of the successive derivatives of the normal probability function. For $K_{0}$ and $K_{1}$,we find, for example,

$$
\begin{aligned}
& K_{0}\left(y+1+x(2 y+1)^{1 / 2}, y\right) \\
& =\frac{1}{(2 y+1)^{1 / 2}} \Phi(x)-\frac{3 y+1}{3(2 y+1)^{2}} \Phi^{(3)}(x) \\
& \quad+\left[\frac{4 y+1}{4(2 y+1)^{5 / 2}} \Phi^{(4)}(x)+\frac{(3 y+1)^{2}}{18(2 y+1)^{7 / 2}} \Phi^{(6)}(x)\right]+O\left(\frac{1}{y^{2}}\right) \\
& K_{1}\left(y+x(2 y)^{1 / 2}, y\right) \\
& =\frac{1}{(2 y)^{1 / 2}} \Phi(x)-\frac{1}{4 y} \Phi^{(3)}(x) \\
& \quad+\frac{1}{4 y(2 y)^{1 / 2}}\left[\Phi^{(4)}(x)+\frac{1}{4} \Phi^{(6)}(x)\right]+O\left(\frac{1}{y^{2}}\right) .
\end{aligned}
$$

Asymptotic series of this type are common in statistics. ${ }^{7}$ They are related to the moments, $\int_{0}^{\infty} x^{m} K_{n}(x, y) d x$, of the function $K_{n}(x, y)$, and may be derived, for example, directly from the Laplace transform. Expansions of each of the functions $K_{n}(x, y), n \geqq 2$, may be obtained in this manner.

\section{Hydrocarbon Research, Inc., New York, N. Y.}

${ }^{7}$ H. Cramer, Mathematical methods of statistics, Princeton, 1946, pp. 227-229. 